\title{
Generalized Energy-Dependent $Q$ Values for Fission
}

R. Vogt

April 1, 2010

Nuclear Data 2010

Jeju, South Korea

April 26, 2010 through April 30, 2010 
This document was prepared as an account of work sponsored by an agency of the United States government. Neither the United States government nor Lawrence Livermore National Security, LLC, nor any of their employees makes any warranty, expressed or implied, or assumes any legal liability or responsibility for the accuracy, completeness, or usefulness of any information, apparatus, product, or process disclosed, or represents that its use would not infringe privately owned rights. Reference herein to any specific commercial product, process, or service by trade name, trademark, manufacturer, or otherwise does not necessarily constitute or imply its endorsement, recommendation, or favoring by the United States government or Lawrence Livermore National Security, LLC. The views and opinions of authors expressed herein do not necessarily state or reflect those of the United States government or Lawrence Livermore National Security, LLC, and shall not be used for advertising or product endorsement purposes. 


\title{
GENERALIZED ENERGY-DEPENDENT $Q$ VALUES FOR FISSION
}

\author{
R. VOGT \\ Lawrence Livermore National Laboratory, Livermore, CA 94550, USA \\ Physics Department, University of California at Davis, Davis, CA 95616, USA \\ E-mail : vogt2@1lnl.gov \\ Received \\ Accepted for Publication
}

We extend Madland's parameterization of the energy release in fission to obtain the dependence of the fission $Q$ value for major and minor actinides on the incident neutron energies in the range $0 \leq E_{n} \leq 20 \mathrm{MeV}$. Our parameterization is based on the actinide evaluations recommended for the ENDF/B-VII.1 release.

KEYWORDS : Nuclear Data, Fission Energy Release, ENDF

\section{INTRODUCTION}

This paper describes the calculation of energydependent fission $Q$ values based on the calculation of the prompt energy release in fission by Madland [1]. This calculation was adopted for use in the LLNL ENDL database in Ref. [2] and then generalized to obtain the prompt fission energy release for all actinides [3]. Here the calculation is further generalized to the total energy release in fission.

There are several stages in a fission event, depending on the time scale. Neutrons and gammas may be emitted at any time during the fission event. While our discussion here is focussed on compound nucleus creation by an incident neutron, similar parameterizations could be obtained for incident gammas or spontaneous fission.

The excited, fissioning compound nucleus of mass $A$ and charge $Z$ is created by the interaction of an incident neutron, $n$, of mass $m_{n}$ and incident energy $E_{n}$, with a target of mass $A-1$ and charge $Z$.

After the compound nucleus has been formed, it has kinetic energy $T$ and excited mass $M^{*}(Z, A)$. We assume binary fission of the compound nucleus into a light, $L$, and a heavy, $H$, fragment. After fission, the excited fragments will decay by neutron and gamma emission. Neutron emission typically occurs first, on a shorter time scale, $10^{-18}-10^{-13}$ $\mathrm{s}$, while the fragment excitation energy is above the neutron separation energy, $S_{n}$. Neutron emission will change the mass number of the initial fragment. Thus if the number of neutrons emitted by the de-excitation of fragment $i$ is denoted as $v_{i}$, the baryon number of product $i$ is

$$
\tilde{A}_{i}=A_{i}-v_{i}
$$

We note that as the fragment $A$ changes, the neutron separation energy may differ between e.g. $A_{i}$ and $A_{i}-1$ so that the number of emitted neutrons is very sensitive to the initial fragment identity. Gamma emission is responsible for the de-excitation of $\tilde{A}_{i}$ to its ground state for excitation energies less than the neutron separation energy over a time scale of $10^{-14}-10^{-7} \mathrm{~s}$. This is the end of the prompt fission energy release. Note that while the $A$ of the fragment has changed, $Z_{i}$ remains the same throughout the prompt stage. Note also that, if the excitation energy is high enough for the excited compound nucleus to emit one or more neutrons before fissioning, these neutrons resulting from multichance fission are also considered to be part of the prompt yield.

After prompt neutron and gamma emission is finished, the residual nuclei are referred to as fission 'products' rather than fragments, a term reserved for the nuclei produced in the binary fission process in Madland's presentation. On a longer time scale, the fission products can $\beta$ decay, changing the charge from $Z_{i}$ to $Z_{i}+1$ but not the mass number $A_{i}$ since the neutron number changes from $N_{i}$ to $N_{i}-1$. Decay electrons and antineutrinos are also emitted in the $\beta$ decay. If the $\beta$-decay is into a state with an excitation energy larger than the neutron separation energy, neutron emission is favored, decreasing the fragment mass number. These neutrons, time-delayed relative to the prompt emission, are thus referred to as delayed neutrons and are accompanied by delayed gamma emission. If the $\beta$-decay is into a state where the excitation energy is less than $S_{n}$, only delayed gamma emission is possible. At the end of the chain of prompt and delayed neutron and gamma emission and $\beta$ decays, the remaining fission products are called secondary products.

We develop a polynomial expansion, up to second order when sufficient information is available, for each of the quantities comprising the fission energy release to create MT $=458$ files for all actinides in the ENDF/B-VII library [4]. We follow the recommendations of Ref. [5] and use the energy deposition for prompt and delayed neutrons from the JENDL Actinoid 2008 database [6,7] proposed for the ENDF/B-VII.1 library. In the remainder of the paper, we describe our model of the energy release and present results 
for several important isotopes: ${ }^{232} \mathrm{Th},{ }^{235} \mathrm{U},{ }^{238} \mathrm{U},{ }^{239} \mathrm{Pu}$ and ${ }^{252} \mathrm{Cf}$.

\section{MODEL DESCRIPTION}

We follow the nomenclature of Sher and Beck [8], comprising nine specific components of the energy release. They are as follows:
EFR fission product kinetic energy;
ENP prompt neutron kinetic energy;
EGP total prompt gamma energy;
END delayed neutron kinetic energy;
EGD total delayed gamma energy;
EB energy released by electrons from $\beta$ decay;
ENU energy lost to neutrinos;
ET sum of all the above components, the total energy release per fission and equal to the fission $Q$ value;
ER effective energy release, the $Q$ value reduced by the energy lost to neutrinos.

To this we introduce, for convenience, two additional terms:

EP prompt energy release in fission;

ED energy released in the latter stages of the fission process, excluding delayed neutron emission.

We parameterize the energy dependence of the above quantities by a second order polynomial where sufficient information is available and a first order polynomial if less is known,

$$
E_{i}\left(E_{n}\right)=c_{0}^{i}+c_{1}^{i} E_{n}+c_{2}^{i} E_{n}^{2},
$$

where $i$ refers to one of the components of the energy release above.

The average fission product kinetic energy for thermal neutrons, $E_{n} \approx 0$, can be obtained through the Coulomb approximation,

$$
\left\langle\operatorname{EFR}\left(E_{n}=0\right)\right\rangle=\alpha \hbar c \frac{Z_{L} Z_{H}}{R_{A_{L}}+R_{A_{H}}+d_{L H}} .
$$

The total fragment kinetic energy is directly proportional to the product of the fragment charges and inversely proportional to the separation of the fragment centers at scission. We take $d_{L H}=1.5 \mathrm{fm}$ and $R_{A}=r_{0} A^{1 / 3}\left(1+2 \beta_{1} / 3\right)$ with $r_{0}=1.16 \mathrm{fm}$ and $\beta_{1}=0.625$ [9]. For actinides up to $\mathrm{Cm}$, we assume that, on average, the heavy fragment has $A_{H}=140$. Above $Z=96$, we increment the average $A$ by 1. The charge of the heavy fragment is obtained assuming $Z_{H}=A_{H}(Z / A)-1 / 2$. The mass and charge of the light fragment is obtained by energy conservation: $A_{L}=A-A_{H}$ and $Z_{L}=Z-Z_{H}$. The average fission product kinetic energy decreases with $E_{n}[1]$. The slope, $c_{1}^{\mathrm{EFR}}$, seems to be a slowly increasing function of $Z$, based on the difference between the ${ }^{239} \mathrm{Pu}$ slope and the average of the ${ }^{235} \mathrm{U}$ and ${ }^{238} \mathrm{U}$ slopes in Ref. [1]. Thus the lighter actinides have a slower decrease in kinetic energy with $E_{n}$ than the heavier ones [3].

Increasing $Z$ with fixed $d_{L H}$ causes $c_{\mathrm{EFR} 0}$ to increase with $A$. One might assume that since fission is more symmetric $\left(Z_{L} \approx Z_{H}, A_{L} \approx A_{H}\right)$ at higher energies, the average
EFR would increase slightly with $E_{n}$ rather than decrease, as implied by the negative slope, $c_{1}^{\mathrm{EFR}}$. However measurements of fragment kinetic energy as a function of $A_{H}$ at fixed $E_{n}$ $[10,11]$ show that $\operatorname{EFR}\left(A_{H}\right)$ is minimized near symmetric fission. This is a general feature of EFR that has been observed a number of times [10-12]. It is a result of several effects. The maximum kinetic energy occurs for values of $A$ where the proton and/or neutron shell is closed $(Z=50$, $N=82$ ). The $A$ values corresponding to the closed shells are away from the region of symmetric fission. Instead there is a dip in the kinetic energy at $A_{L} \approx A_{H}$ thought to be due to the greater deformation of the pre-fragments at the symmetry point, leading to a larger $d_{L H}$. Indeed, while we have modeled $c_{1}^{\mathrm{EFR}}$ on the change in $Z$, we could as well have modeled it based on increasing $d_{L H}$ with $E_{n}$.

Once we have selected the identities of the average fragments, we can calculate the $Q$ value for the fission reaction, $n+{ }^{A} Z \rightarrow{ }^{A_{L}} Z_{L}+{ }^{A_{H}} Z_{H}$, as the difference in the mass excess for the nuclei involved in the reaction,

$$
Q=M_{A}+m_{n}-M_{A_{L}}-M_{A_{H}} .
$$

The prompt neutron, prompt gamma and delayed neutron energy depositions compiled in the databases for a given incident neutron energy, $E_{n}$, is the product of the average multiplicity of the outgoing particle, $\mu_{i}\left(E_{n}\right)$, and the average energy of a single particle,

$$
\left\langle E_{i}\right\rangle=\frac{\int d E_{i} E_{i} P\left(E_{n}, E_{i}\right)}{\int d E_{i} P\left(E_{n}, E_{i}\right)} .
$$

The upper and lower limits of the integrals in Eq. (5) are the maximum and minimum possible energies of the outgoing particle.

Where there are sufficient energy points to discern the difference between linear and quadratic fits to the outgoing energies, as is the case for prompt neutrons and some of the delayed neutrons, a quadratic fit is performed using the CERN ROOT software package which gives both the best fit and the fit parameter uncertainty. Otherwise, a linear fit is used. When only two energy values are given for a range of incident energies, typical when six-energy group measurements are available for delayed neutrons, the centroids of the bins are used to calculate the slope and intercept. The edges of the bins are used to estimate the uncertainties in the slope and intercept.

The prompt gamma energy deposition information included in the databases is rather sparse. The behavior is either missing or somewhat unphysical. The increase with $E_{n}$ seen by Madland for ${ }^{235} \mathrm{U},{ }^{238} \mathrm{U}$ and ${ }^{239} \mathrm{Pu}$ [1] is not found in the databases. We thus use the EGP results tablulated in ENDL99 [13] when available and choose the behavior of ${ }^{232} \mathrm{U}$ for a generic prompt gamma energy deposition.

Since there are so few data available, it is not clear how 'generic' the dependence actually is. One might naively expect that more energy is taken away by gamma emission as the mass of the compound nucleus and the incident neutron energy increase. However, prompt gamma emission comes only after the fission product excitation energy drops 
below the neutron separation energy, a few MeV. Therefore the fragment is at a similar level of excitation when prompt gamma emission begins, regardless of the incident neutron energy. Consequently, the separation energy should thus provide a lower bound on EGP, independent of $E_{n}$, and its dependence on incident energy may indeed be slow. A stronger variation with $A$ may, however, be expected since the neutron separation energy depends on the individual fragment pairing energy.

The decay energy deposition from betas, neutrinos and delayed gammas is largely unknown. We determine its contribution at $E_{n} \approx 0$ from the total $Q$ value, Eq. (4); the prompt energy deposition; and the energy deposited by delayed neutrons. The prompt energy deposition for $E_{n} \approx 0$ is

$$
\mathrm{EP}(0)=\mathrm{EFR}(0)+\mathrm{ENP}(0)+\mathrm{EGP}(0) .
$$

The corresponding decay energy, which, by definition, does not include delayed neutrons, is then

$$
\mathrm{ED}(0)=Q(0)-\mathrm{EP}(0)-\mathrm{END}(0) .
$$

Sher and Beck found that, within the nuclei they examined, the fraction of the decay energy going to betas, neutrinos and delayed gammas was independent of the identity of the compound nucleus [8]. Since the characteristics of $\beta$ decay are unique to the properties of the fission products rather than the fissioning compound nucleus itself, this result is not surprising. We assume that the ratios [8]

$$
\begin{aligned}
\frac{\operatorname{EB}(0)}{\operatorname{ED}(0)} & =0.3015 \pm 0.0010, \\
\frac{\operatorname{ENU}(0)}{\operatorname{ED}(0)} & =0.4053 \pm 0.0015, \\
\frac{\operatorname{EGD}(0)}{\operatorname{ED}(0)} & =0.2932 \pm 0.0015,
\end{aligned}
$$

hold for all the actinides considered. The energy dependence is largely unknown. A "best guess" is included in the ENDF/B-VII manual [14],

$$
\begin{aligned}
c_{1}^{\mathrm{EB}} & =-0.075, \\
c_{1}^{\mathrm{ENU}} & =-0.100, \\
c_{1}^{\mathrm{EGD}} & =-0.075 .
\end{aligned}
$$

We assign a $10 \%$ uncertainty to these slopes, a conservative estimate. There is no justification for including a quadratic term.

The contributions to the energy release from EFR, ENP, EGD, END, EB and ENU are shown in Fig. 1 for our chosen set of isotopes. We have already discussed the decrease of EFR with energy. The prompt neutron energy increases because the prompt neutron multiplicity increases with the increased fragment excitation energy possible at higher $E_{n}$. This increase is not typically monotonic because, at sufficiently high energy, it becomes more probable for the excited compound nucleus to emit one or more neutrons before fission, known as multi-chance fission. These neutrons
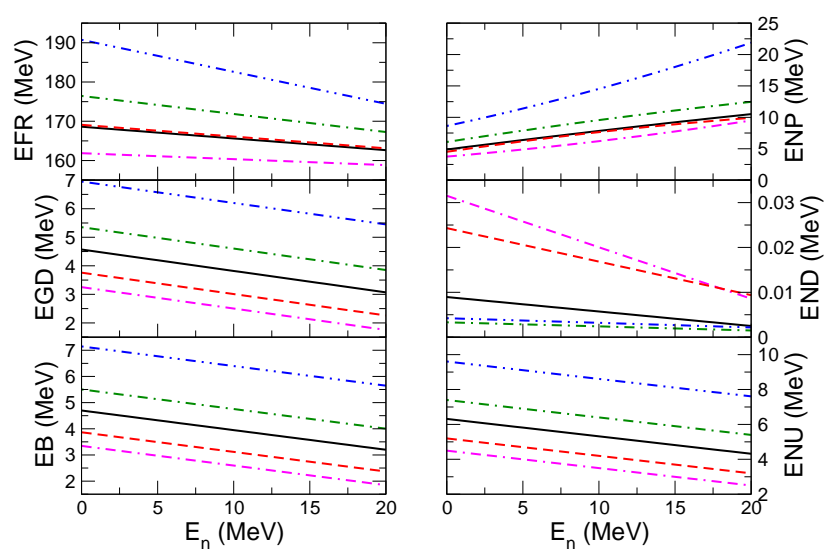

Fig. 1. Contributions to the total energy release from fission for ${ }^{232} \mathrm{Th}$ (dot-dot-dashed), ${ }^{235} \mathrm{U}$ (solid), ${ }^{238} \mathrm{U}$ (dashed), ${ }^{239} \mathrm{Pu}$ (dot-dashed) and ${ }^{252} \mathrm{Cf}$ (dot-dash-dashed) as a function of incident neutron energy. The left-hand column shows EFR (top), EGD (center) and EB (bottom). The right-hand column shows ENP (top), END (center) and ENU (bottom).

contribute to the total prompt neutron multiplicity. They are also likely one reason for the slow decrease of the energy release by the decay products shown in Fig. 1 since emission of a neutron prior to fission reduces the excitation energy of the fissioning daughter, likely also reducing the probability of $\beta$ decay.

The total energy release is then

$$
\mathrm{ET}\left(E_{n}\right)=\mathrm{EP}\left(E_{n}\right)+\operatorname{ED}\left(E_{n}\right)+\operatorname{END}\left(E_{n}\right)-E_{n},
$$

where we subtract the energy of the incident neutron since it is not part of the energy release. The effective energy release, ER, does not include ENU which is lost since the neutrinos depart the system without interacting and therefore do not deposit any energy,

$$
\operatorname{ER}\left(E_{n}\right)=\operatorname{ET}\left(E_{n}\right)-\operatorname{ENU}\left(E_{n}\right) .
$$

\section{SAMPLE RESULTS}

The resulting new representation, including uncertainties, is incoporated into the MT $=458$ as follows for each component of the polynomial in Eq. (2), denoted by the subscript $m$ here:

$$
\begin{array}{cccccc}
c_{m}^{\mathrm{EFR}} & \Delta c_{m}^{\mathrm{ER}} & c_{m}^{\mathrm{ENP}} & \Delta c_{m}^{\mathrm{ENP}} & c_{m}^{\mathrm{END}} & \Delta c_{m}^{\mathrm{END}} \\
c_{m}^{\mathrm{EGP}} & \Delta c_{m}^{\mathrm{EGP}} & c_{m}^{\mathrm{EGD}} & \Delta c_{m}^{\mathrm{EGD}} & c_{m}^{\mathrm{EB}} & \Delta c_{m}^{\mathrm{EB}} \\
c_{m}^{\mathrm{ENU}} & \Delta c_{m}^{\mathrm{ENU}} & c_{m}^{\mathrm{ER}} & \Delta c_{m}^{\mathrm{ER}} & c_{m}^{\mathrm{ET}} & \Delta c_{m}^{\mathrm{ET}}
\end{array}
$$

This set of lines is repeated one time more than the maximum polynomial order.

The prompt energy release,

$$
\mathrm{ER}_{\text {prompt }}\left(E_{n}\right)=\operatorname{EP}\left(E_{n}\right)-E_{n},
$$

is shown as a function of $E_{n}$ in Fig. 2. It decreases more slowly with incident energy than the total energy release, 


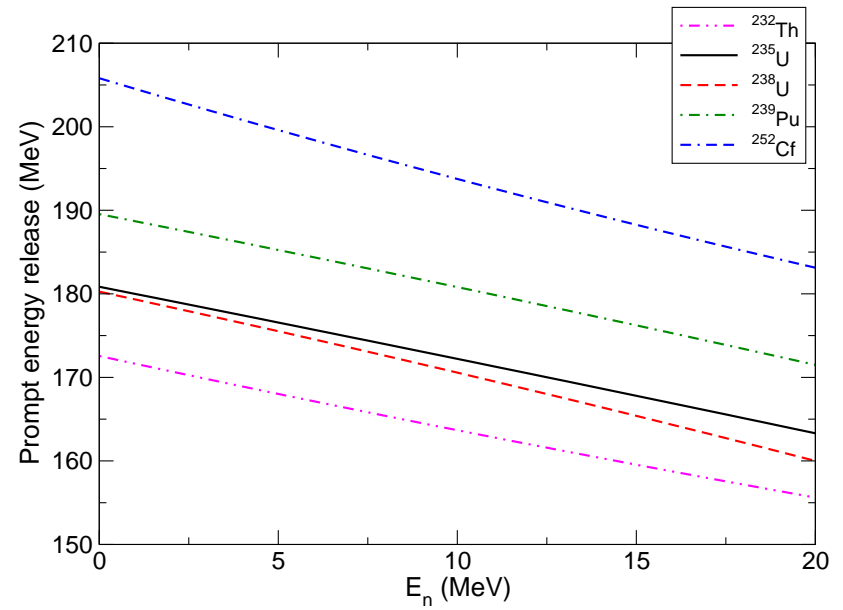

Fig. 2. The prompt energy release from fission, EP, for ${ }^{232} \mathrm{Th}$ (dot-dot-dashed), ${ }^{235} \mathrm{U}$ (solid), ${ }^{238} \mathrm{U}$ (dashed), ${ }^{239} \mathrm{Pu}$ (dot-dashed) and ${ }^{252} \mathrm{Cf}$ (dot-dash-dashed) as a function of $E_{n}$.

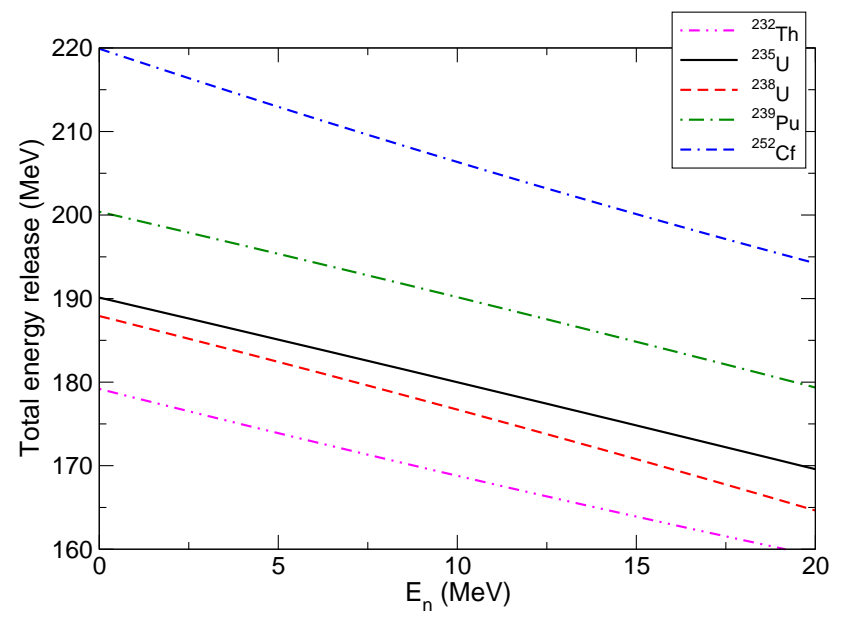

Fig. 3. The total energy release from fission, ER, for ${ }^{232} \mathrm{Th}$ (dot-dot-dashed), ${ }^{235} \mathrm{U}$ (solid), ${ }^{238} \mathrm{U}$ (dashed), ${ }^{239} \mathrm{Pu}$ (dot-dashed) and ${ }^{252} \mathrm{Cf}$ (dot-dash-dashed) as a function of $E_{n}$.

$\mathrm{ER}$, shown in Fig. 3. While ER is larger than $\mathrm{ER}_{\text {prompt }}$ at $E_{n} \approx 0$ due to the decay energy, it decreases faster because we have assumed that the decay energy contribution decreases with $E_{n}$.

\section{ACKNOWLEDGMENTS}

We thank D. A. Brown for discussions. This work was performed under the auspices of the U.S. Department of Energy by Lawrence Livermore National Laboratory under Contract DE-AC52-07NA27344 and was also supported in part by the National Science Foundation Grant NSF PHY0555660 .

\section{REFERENCES}

[1] D. Madland, "Total Prompt Energy Release in the NeutronInduced Fission of ${ }^{235} \mathrm{U},{ }^{238} \mathrm{U}$ and ${ }^{239} \mathrm{Pu}$," Nucl. Phys. A, 772, 113 (2006).

[2] R. Vogt, B. Beck, D. A. Brown, F. Daffin and J. Hedstrom, "Implementation of Energy-Dependent $Q$ Values for Fission," UCRL-TR-234617, Lawrence Livermore National Laboratory (2007).

[3] R. Vogt, "Generalized Energy-Dependent Fission $Q$ Values," LLNL-TR-407620, Lawrence Livermore National Laboratory (2008).

[4] M. B. Chadwick et al., "ENDF/B-VII.0: Next Generation Evaluated Nuclear Data Library for Nuclear Science and Technology," Nucl. Data Sheets 107, 2931 (2006).

[5] D. A. Brown, "Review of JENDL Actinoid File 2008," LLNL-TR-419387, Lawrence Livermore National Laboratory (2009).

[6] O. Iwamoto, T. Nakagawa, N. Otuka, S. Chiba, K. Okumura, G. Chiba, T. Ohsawa and K. Furutaka, "JENDL Actinoid File 2008," J. Nucl. Sci. Tech. 46, 510 (2009).

[7] O. Iwamoto, T. Nakagawa, N. Otuka, S. Chiba, K. Okumura and G. Chiba, "JENDL Actinoid File 2008 and Plan of Covariance Evaluation," Nucl. Data Sheets 109, 2885 (2008).

[8] R. Sher and C. Beck, "Fission Energy Release for 16 Fissioning Nuclides," EPRI-NP-1771 (1981).

[9] K.-H. Schmidt, S. Steinhaüser, C. Böckstiegel, A. Grewe, A. Heinz, A. R. Junghans, J. Benlliure, H.-G. Clerc, M. deJong, J. Müller, M. Pfützner and B. Voss, "Relativistic Radioactive Beams: A New Access to Nuclear Fission Studies," Nucl. Phys. A 665, 221 (2000).

[10] H. W. Schmitt, J. H. Neiler and F. J. Walter, "Fragment Energy Correlation Measurements for ${ }^{252} \mathrm{Cf}$ Spontaneous Fission and ${ }^{235} \mathrm{U}$ Thermal-Neutron Fission," Phys. Rev. 141, 1146 (1966).

[11] J. N. Neiler, F. J. Walter and H. W. Schmitt, "Fission Fragment Energy Correlation Measurements for the Thermal Neutron Fission of ${ }^{239} \mathrm{Pu}$ and ${ }^{241} \mathrm{Pu}$," Phys. Rev. 149, 894 (1966).

[12] J. C. D. Milton and J. S. Fraser, "Time of Flight Fission Studies on ${ }^{233} \mathrm{U},{ }^{235} \mathrm{U}$ and ${ }^{239} \mathrm{Pu}$," Can. J. Phys. 40, 1626 (1962); "Prompt Fission Yields and Total Kinetic Energy Behavior from Time of Flight Measurements," Phys. Rev. Lett. 7, 67 (1961).

[13] B. Beck, G. W. Hedstrom, T. S. Hill, A. A. Marchetti and D. P. McNabb, "ASCII format specifications for the Evaluated Nuclear Data Libraries (ENDL)", UCRL-TM-218475 (2006).

[14] M. Herman and A. Trkov, editors, "ENDF-6 Formats Manual: Data Formats and Procedures for the Evaluated Nuclear Data File ENDF/B-VI and ENDF/B-VII", CSWEG Document ENDF-102, Report BNL-90365-2009, Brookhaven National Laboratory (2009). 